\title{
Fine needle aspiration cytology (FNAC) of nerves in leprosy
}

\author{
M. VIJAIKUMAR, MARIETTE D'SOUZA, \\ SURENDRA KUMAR* \& BHAWANA BADHE* \\ Departments of Dermatology and STD and *Pathology, Jawaharlal \\ Institute of Postgraduate Medical Education and Research \\ (JIPMER), Pondicherry 605 006, India
}

\author{
Accepted for publication 5 April 2001
}

\begin{abstract}
Summary Leprosy is primarily a disease of the peripheral nerves and a technique that is simpler than nerve biopsy is required to evaluate nerve involvement, especially in pure neuritic (PN) leprosy. This study was designed to evaluate the role of FNAC of the nerve in the diagnosis and classification of leprosy. A prospective study was carried out on 25 patients with clinically active leprosy and at least one thickened peripheral sensory nerve. Nerve aspirates were evaluated by May-GrunwaldGiemsa and Fite's staining. Lepromin test, slit skin smears (SSS), skin biopsies (except PN cases) and nerve biopsies were performed and compared with FNAC. FNAC of nerve from 23 cases $(92 \%)$ yielded diagnostic aspirates. Acid fast bacilli were observed in six cases by FNAC. FNAC and nerve pathology were equally comparable with the other parameters evaluated. Based on the results, cytological criteria were developed for interpreting nerve aspirates and the cases were classified as paucibacillary (18), BB (2), BL (2), LL (1) and non-diagnostic (2). All PN cases showed diagnostic paucibacillary type cytology. FNAC of the nerve yields diagnostic aspirates in leprosy comparable with nerve pathology and the proposed cytological criteria may be useful in classification of nerve aspirates.
\end{abstract}

\section{Introduction}

Although leprosy is primarily a disease of the peripheral nerves, the main criteria for diagnosis and classification are related to skin parameters such as slit skin smears (SSS) and skin biopsies. ${ }^{1-3}$ Several studies have shown discrepancies between skin and nerve histopathology, with a higher bacterial load in the peripheral nerves when compared to the skin. ${ }^{4-8}$ In addition, pure neuritic (PN) leprosy, which involves the nerves alone without any skin changes, is a definite entity recognized in the Indian classification of leprosy. ${ }^{9}$ The diagnosis of this form of leprosy can be confirmed only by a nerve biopsy. ${ }^{2}$

* Correspondence to: M. D’Souza (e-mail :skverma60@satyam.net.in) 
Hence, a technique that is simpler than nerve biopsy is required to evaluate nerve involvement, especially in pure neuritic leprosy. Cytology is widely accepted as a diagnostic procedure for a large variety of malignant and inflammatory lesions. ${ }^{10}$ Fine needle aspiration cytology (FNAC) of skin lesions and lymph nodes has already been shown to be useful in the diagnosis and classification of leprosy..$^{10,11}$ Theuvenet et al. ${ }^{12}$ and Jayaseelan et al. ${ }^{13}$ have shown that FNAC of the nerve could yield adequate material for the diagnosis of pure neuritic leprosy. This study was designed to evaluate the role of FNAC of the peripheral sensory nerve in the diagnosis and classification of leprosy by comparing the aspirate with nerve pathology by biopsy.

\section{Materials and methods}

Twenty-five consecutive patients with a clinical diagnosis of leprosy and having at least one thickened peripheral sensory nerve were included in the study. Informed consent for performing FNAC and biopsy of the nerves was obtained from all the patients. The study population included patients attending the leprosy clinic of our department and those referred from other specialities of our institute with features of peripheral nerve involvement.

Slit-skin smears for acid-fast bacilli (AFB) from lesional skin, ear lobules and normal skin and lepromin test (lepromin-A) were done in all the cases. Skin biopsies were obtained from the suggestive lesions (except pure neuritic cases).

Fine needle aspiration was done from the peripheral sensory cutaneous nerves, viz. radial cutaneous nerve (15), cutaneous nerves of forearm (5), sural nerve (4) and superficial peroneal nerve (1). A disposable $10 \mathrm{ml}$ plastic syringe fitted with a $21 \mathrm{G}$ needle was used. We used a 'hand held' method rather than a syringe holder to create negative pressure. The nerve was fixed between the thumb and the index finger of one hand, and with the other, the needle was inserted into the nerve as parallel to the nerve as possible and aspiration was performed by creating negative pressure. Sensory function and tenderness of the aspirated nerve were assessed clinically on the day of aspiration and 1 week after the procedure. Nerve biopsies were taken in all the cases from the same nerve 1 week after the FNAC procedure.

The biopsies were studied in haematoxylin and eosin (H\&E) stained sections, and modified Fite-Faraco staining was used to evaluate the bacteriological index (BI) according to the Ridley's logarithmic scale. ${ }^{1}$ The histological criteria as described by Ridley ${ }^{14}$ for skin biopsies and Ridley and Ridley ${ }^{15}$ for nerve biopsies were used to classify the cases along the Ridley-Jopling classification. The smears for cytology were air dried and cytological assessment was done by May-Grunwald-Giemsa (MGG) staining and AFB status assessed by modified Fite's staining.

\section{Results}

The age of our patients ranged from 21 to 59 years (mean 38 years), with 20 men and five women. The duration of the presenting clinical feature ranged from 2 weeks to 4 years. Based on the clinical criteria according to Ridley-Jopling classification ${ }^{3}$ and using the criteria of 
Table 1. Correlation of nerve pathology and nerve cytology. $\mathrm{I}=$ indeterminate, $\mathrm{NS}=$ non-specific, Ep $=$ epithelioid cell, $\mathrm{Lc}=$ lymphocyte, $\mathrm{Ma}=$ macrophage, $\mathrm{PMN}=$ neutrophil, $\mathrm{GC}=$ giant cell, $\mathrm{AFB}=$ acid fast bacilli, $\mathrm{GA}=\mathrm{good}$ aspirate

\begin{tabular}{|c|c|c|c|c|c|c|c|c|c|c|c|c|c|c|c|}
\hline \multirow[b]{3}{*}{ Nerve pathology } & \multicolumn{15}{|c|}{ Nerve cytology } \\
\hline & \multicolumn{3}{|c|}{ Granuloma } & \multirow[b]{2}{*}{$\mathrm{Ma}$} & \multirow{2}{*}{$\begin{array}{l}\text { Ep } \\
++\end{array}$} & \multirow[b]{2}{*}{+} & \multirow{2}{*}{$\begin{array}{c}\mathrm{Lc} \\
++\end{array}$} & \multirow[b]{2}{*}{+} & \multirow{2}{*}{$\begin{array}{l}\mathrm{Ma} \\
++\end{array}$} & \multirow[b]{2}{*}{+} & \multirow{2}{*}{$\begin{array}{c}\text { PMN } \\
++\end{array}$} & \multirow[b]{2}{*}{+} & \multirow{2}{*}{$\begin{array}{c}\text { GC } \\
+\end{array}$} & \multirow[b]{2}{*}{ GA } & \multirow{2}{*}{$\begin{array}{c}\text { AFB } \\
+\end{array}$} \\
\hline & + & - & Ep & & & & & & & & & & & & \\
\hline $\mathrm{TT}(2)$ & 2 & - & 2 & - & 2 & - & - & 2 & - & - & - & - & - & 2 & - \\
\hline BT(14) & 6 & 8 & 6 & - & 6 & 8 & 7 & 7 & - & - & - & 5 & 4 & 14 & 2 \\
\hline $\mathrm{BB}(2)$ & 1 & 1 & - & 1 & 1 & 1 & - & 2 & 1 & 1 & - & - & - & 2 & 1 \\
\hline $\mathrm{BL}(2)$ & - & 2 & - & - & - & - & 2 & - & - & 2 & - & 1 & - & 2 & 2 \\
\hline $\mathrm{LL}(2)$ & - & 2 & - & - & - & - & - & 2 & 1 & - & - & - & - & 1 & 1 \\
\hline $\mathrm{I}(2)$ & - & 2 & - & - & - & 1 & 1 & 1 & - & - & - & 1 & - & 1 & - \\
\hline NS(1) & 1 & - & 1 & - & 1 & - & 1 & - & - & - & - & - & 1 & 1 & - \\
\hline
\end{tabular}

the Indian classification of leprosy for pure neuritic leprosy, ${ }^{9}$ we classified our cases clinically as BT (12), BB (1), BL (2), LL (2) and pure neuritic (8).

\section{CYTOLOGY OF NERVE ASPIRATES}

Out of the 25 cases, good aspirates with adequate cellularity enabling classification along the Ridley-Jopling classification were obtained in 23 cases (92\%). AFB were obtained in six cases $(24 \%)$ by nerve aspiration which correlated with nerve biopsy, except in one LL case, which was negative for AFB by cytology (Table 1).

A cohesive collection of epithelioid cells or macrophages in the cytological smear was defined as a granuloma (Figure 1). The granulomas observed were epithelioid (9) and macrophage (1). Collections of lymphocytes alone were observed in two cases. By comparing the cellular features and $\mathrm{BI}$ of nerve aspirates with the histopathology and BI of nerve

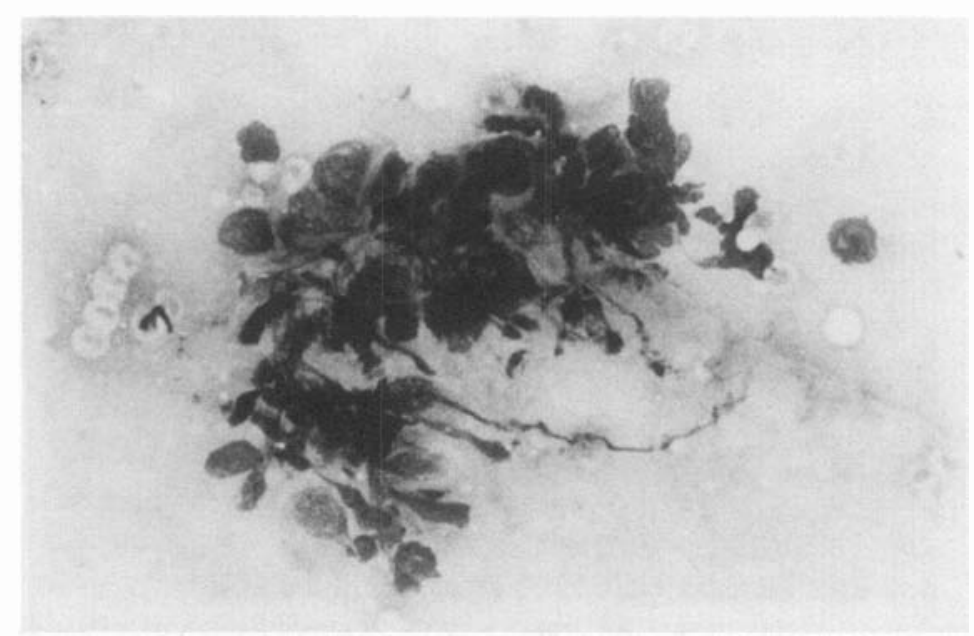

Figure 1. Cohesive epithelioid cell granuloma in a case of pure neuritic leprosy (FNAC nerve, MGG $\operatorname{stain} \times 250$ ). 


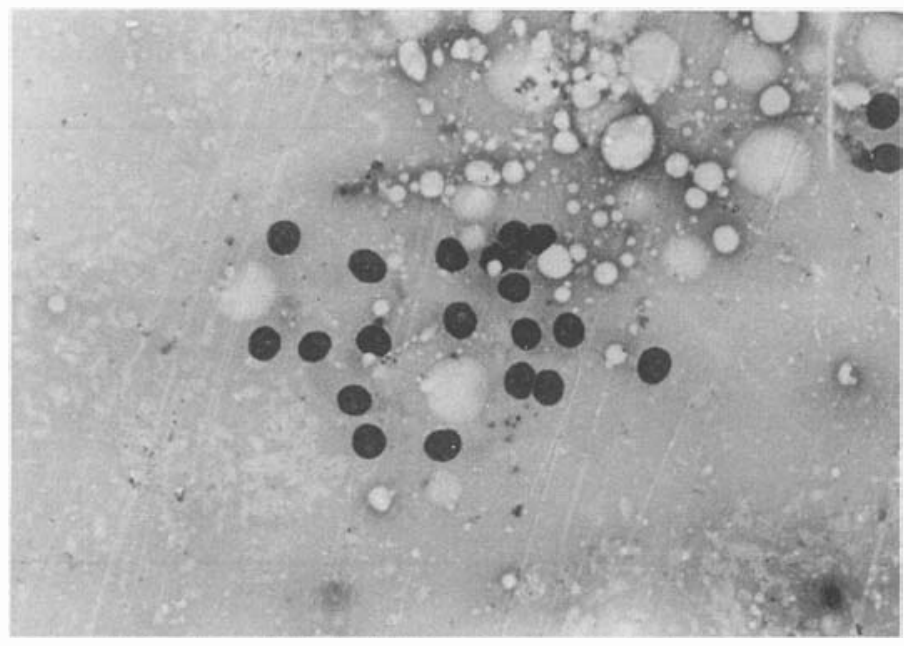

Figure 2. Lymphocytes in a case of tuberculoid leprosy (FNAC nerve, MGG stain $\times 250$ ).

biopsies (Table 1) a set of criteria was devised for interpreting the cytology of nerve aspirates as follows.

Paucibacillary $(P B)$ pole (consisting of indeterminate, TT and BT)

- Good cellular aspirate

- Cohesive epithelioid cell granuloma or lymphocytic cell collection (Figures 1 and 2).

- Predominantly epithelioid cells with predominant to moderate number of lymphocytes (Figures 1 and 3)

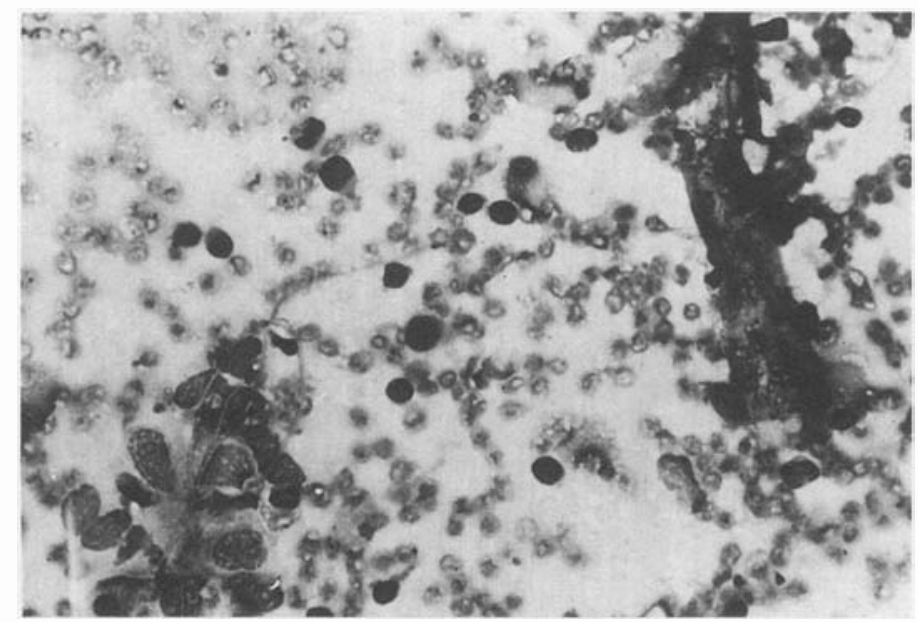

Figure 3. Lymphocytes, epitheliod cells, nerve fibre in a case of tuberculoid leprosy (FNAC nerve, MGG stain $\times 500)$. 
- Occasional giant cells and neutrophils

- $\mathrm{BI}=$ zero to $1+$

\section{Borderline borderline $(B B)$}

- Fair cellular aspirate

- Mixed cellularity of predominantly non-foamy macrophages, moderate number of epithelioid cells and lymphocytes.

- Macrophage granuloma

- $\mathrm{BI}=2+$ to $3+$

Borderline lepromatous (BL)

- Fair cellular aspirate

- Predominantly lymphocytes and moderate number of foamy macrophages

- $\mathrm{BI}=4+$ to $5+$

\section{Lepromatous leprosy (LL)}

- Fair to poor cellular aspirate

- Predominantly foamy macrophages and few lymphocytes

- $\mathrm{BI}=6+$

Based on the proposed criteria, cytological classification of the 23 cases with good aspirate was: PB (18), BB (2), BL (2) and LL (1). In two cases the aspirate was non-diagnostic. No cases with nerve abscesses were observed in the study. However, caseous necrosis was observed in three cases both in nerve histopathology and FNAC.

\section{PURE NEURITIC CASES}

By nerve biopsy, the cases in our study were classified as TT (2), BT (5), and indeterminate (1). All the eight cases of pure neuritic leprosy yielded adequate aspirates showing PB type cytology. Epithelioid cell granulomas were observed in three cases and a BI of 1+ was seen in one case.

\section{CORRELATION OF NERVE PATHOLOGY AND CYTOLOGY}

Classification of cases by nerve biopsy and nerve cytology was comparable in 22 cases ( $88 \%$ concordance). One case with LL pathology in the nerve biopsy yielded a non-specific aspirate and another case with indeterminate type of pathology showed a diagnostic PB type cytology (Tables 1 and 2).

Among the non-pure neuritic cases, concordance between clinical diagnosis and nerve cytology was seen in 14 cases $(82.4 \%)$ and concordance between skin biopsy and nerve cytology was seen in 12 cases $(70.6 \%)$. The concordance percentages between clinical diagnosis and nerve biopsy and that between skin biopsy and nerve biopsy were also 82.4 and $70.6 \%$, respectively. 
Table 2. Grading across Ridley-Jopling classification (excluding pure neuritic cases). $\mathrm{I}=$ indeterminate, $\mathrm{NS}=$ non-specific

\begin{tabular}{lrrrrrrr}
\hline & I & TT & BT & BB & BL & LL & NS \\
\hline Clinical diagnosis & - & - & 12 & 1 & 2 & 2 & - \\
Skin pathology & 2 & 1 & 8 & 1 & 2 & 2 & 1 \\
Nerve pathology & 1 & - & 9 & 2 & 2 & 2 & 1 \\
Nerve cytology & 10 & & & 2 & 2 & 1 & 2 \\
\hline
\end{tabular}

\section{BACTERIOLOGICAL INDEX (EXCLUDING PURE NEURITIC CASES)}

Four cases showed BI of 3+ to 6+ by SSS, skin biopsy and nerve biopsy, but one was negative by FNAC. In cases with $\mathrm{BI}$ of $1+$ to $2+$, nerve biopsy and FNAC were positive for AFB in two cases, while skin biopsy was positive in only one of these and SSS was negative in both (Table 3).

\section{COMPLICATIONS OF FNAC OF THE NERVE}

Tenderness at the aspiration site was observed in 13 cases immediately after the procedure, but none of them had tenderness 7 days after aspiration. None of the patients had significant increase in sensory loss of region supplied by the aspirated nerve 7 days after the procedure.

\section{Discussion}

FNAC is widely accepted as a diagnostic procedure for several malignant and inflammatory lesions. ${ }^{10,13}$ The procedure is simple and can be repeated a second or third time if needed. The efficacy of this procedure in Hansen's disease has, however, not been exploited fully, though, slit skin smears have been conventionally used in leprosy to assess the bacteriological and morphological indices. Also, Ziehl-Neelsen staining does not provide adequate morphological details of the cell types. ${ }^{10}$ Singh et al. ${ }^{10}$ have shown that MGG staining complemented Ziehl-Neelsen staining in skin smears and yielded information almost comparable to skin histopathology. Theuvenet et al. ${ }^{12}$ and Jayaseelan et al. ${ }^{13}$ have shown that adequate aspirates could be obtained from nerves aiding in the diagnosis of pure neuritic leprosy.

Table 3. Bacteriological index (excluding pure neuritic cases). $\mathrm{BI}=$ bacteriological index, SSS = slit skin smear

\begin{tabular}{|c|c|c|c|c|}
\hline \multirow{2}{*}{$\begin{array}{l}\text { skin smear } \\
\text { BI }\end{array}$} & \multicolumn{4}{|c|}{ Number of cases $(\%)$} \\
\hline & SSS & Skin biopsy & Nerve biopsy & FNAC \\
\hline 0 & $13(76 \cdot 5)$ & $12(70 \cdot 5)$ & $11(64 \cdot 7)$ & $12(70 \cdot 5)$ \\
\hline $1+$ to $2+$ & 0 & $1(5 \cdot 9)$ & $2(11.8)$ & $2(11.8)$ \\
\hline $3+$ to $6+$ & $4(23 \cdot 5)$ & $4(23 \cdot 5)$ & $4(23 \cdot 5)$ & $3(17.6)$ \\
\hline Total & 17 & 17 & 17 & 17 \\
\hline
\end{tabular}


This is the only large series in which the cytological findings of nerve aspirates have been compared with the histopathology of the nerves and by analysing these, a set of criteria was devised to classify the cytological smears. Differentiation between indeterminate, TT and BT forms was difficult in cytological smears, which were grouped together as PB leprosy. In the PB pole, cohesive epithelioid cells and lymphocytes were observed. Towards the lepromatous pole, no cohesion of cells was observed. It was observed that the cellular characteristics were more useful in $\mathrm{PB}$ pole and $\mathrm{BB}$ type, and that the $\mathrm{BI}$ was more useful in the lepromatous pole.

The nerve aspirate was negative for AFB in one LL case with non-specific cytology. This may be explained by the associated fibrosis of the nerve in the lepromatous pole of the disease. In cases with lower BI $(1+$ to $2+)$, two cases showed AFB positivity in nerve aspirates but both were negative in slit skin smears. These two cases of BT leprosy would have been classified as PB by the WHO case definition, ${ }^{16}$ whereas in fact, they were multibacillary by nerve cytology.

All the eight cases diagnosed as pure neuritic leprosy yielded diagnostic nerve aspirates and were classified as PB type of leprosy based on cytology with one of the cases showing AFB positivity. In the study by Theuvenet et al. ${ }^{12}$ seven of the 11 cases of suspected pure neuritic leprosy showed multiple AFB in the nerve aspirate. No cytological evaluation was done in that study. In the study by Jayaseelan et al., ${ }^{13} 18$ of 27 cases were diagnosed as pure neuritic leprosy based on cytology of nerve aspirate but only three showed AFB positivity. Hence, we recommend the use of both cytological and AFB evaluation of the nerve aspirates. In both the previous studies published on FNAC of the nerve, aspiration of motor nerves was also done safely. ${ }^{12,13}$ Though no iatrogenic sensory loss was noticed in the superficial sensory nerves, the use of FNAC on motor nerves requires caution and further standardization of the technique.

When nerve pathology and nerve cytology were separately compared with the clinical diagnosis and the skin pathology, equal concordance was observed. A nerve aspirate with cytology as described in the devised criteria or AFB positivity is diagnostic as seen by the 92\% positive aspirates in this study. However, a negative aspirate does not entirely rule out leprosy. Hence FNAC, being a simpler, quicker and less invasive technique, can be attempted on the nerve before deciding on a nerve biopsy. This would be particularly useful when pure neuritic leprosy is suspected.

\section{References}

1 Jopling WH, McDougall AC. Diagnostic tests. In: Handbook of Le prosy, 5th edn. CBS Publishers \& Distributors, New Delhi, 1996; 58-65.

2 Pfaltzgraff RE, Ramu G. Clinical aspects. In: Hastings RC (ed) Leprosy, 2nd edn. Churchill Livingstone, Edinburgh, 1994; 237-287.

${ }^{3}$ Ridley DS, Jopling WH. Classification of leprosy according to immunity. A five group system. Int J Lepr, 1996; 34: $255-273$.

4 Srinivasan H, Rao KS, Iyer CGS. Discrepancy in the histopathological features of leprosy lesions in the skin and peripheral nerve. Lepr Ind, 1982; 54: 275-286.

5 Nilsen R, Mengistu G, Reddy BB. The role of nerve biopsies in the diagnosis and management of leprosy. Lepr Rev, 1989; 60: 28-32.

${ }^{6}$ Kaur S, Sharma VK, Basak P et al. Concurrent skin and nerve histology in leprosy and its role in the classification of leprosy. Lepr Rev, 1993; 64: 110-116.

7 Kumar SKA, Reddy BSN, Ratnakar C. Correlation of skin and nerve histopathology in leprosy. Lepr Rev, 1996; 67: 119-125.

8 Ponnighaus JM, Lienhardt C, Lucas S et al. Comparison of bacillary indexes in slit-skin smears, skin and nerve biopsies; a study from Malawi. Int J Lepr, 1997; 65: 211-216. 
9 Dharmendra. Classifications of leprosy. In: Hastings RC (ed) Leprosy, 2nd edn. Churchill Livingstone, Edinburgh, 1994; 179-192.

10 Singh N, Bhatia A, Gupta K, Ramam M. Cytomorphology of leprosy across the Ridley-Jopling spectrum. Acta Cytol, 1996; 40: 719-723.

11 Singh N, Bhatia A, Arora VK, Bhattacharya SN. Fine needle aspiration cytology of lepromatous leprosy. Lepr Rev, 1998; 69: 145-150.

12 Theuvenet WJ, Miyazaki N, Roche P, Shrestha I. Cytological needle aspiration for the diagnosis of pure neural leprosy. Ind J Lepr, 1996; 68: 109-112.

13 Jayaseelan E, Shariff S, Rout P. Cytodiagnosis of primary neuritic leprosy. Int J Lepr, 1999; 67: 429-434.

14 Ridley DS. Skin biopsy in leprosy, 3rd edn. Documenta Geigy, Basle, 1990; 41-52.

15 Ridley DS, Ridley MJ. Classification of nerves is modified by the delayed recognition of M. leprae. Int J Lepr, 1986; 54: 596-605.

16 WHO Expert Committee on Leprosy, Seventh Report. WHO Technical Report Series 874, World Health Organisation, Geneva, 1997. 